\title{
La educación ambiental en ASADAS de Sabanilla, Carrizal y Santiago de Palmares, Alajuela, Costa Rica
}

\section{Environmental Education in ASADAS in Sabanilla, Carrizal and Santiago de Palmares, Alajuela, Costa Rica}

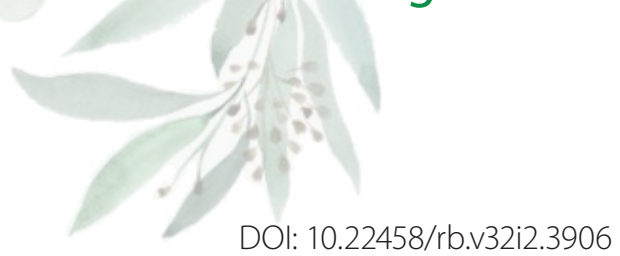

Recibido - Received: 19/10/2021 / Corregido - Revised:09/11/2021 / Aceptado - Accepted: 24/11/2021

\section{RESUMEN}

Se investigó el proceso de educación ambiental (EA) desarrollado por tres Asociaciones Administradoras de Sistemas de Acueductos y Alcantarillados Sanitarios (ASADAS). En este trabajo se describen las actividades de EA realizadas por las mencionadas ASADAS que, además de suministrar agua potable, deben velar por la protección del recurso hídrico. El propósito de la publicación es informar sobre la función que cumplen las ASADAS tanto en la protección del recurso hídrico, como en la conservación de la biodiversidad. La información es útil para mejorar lo que las ASADAS estudiadas han hecho hasta el momento y promover la EA en otras asociaciones. El estudio requirió revisión documental, entrevistas semiestructuradas y la aplicación de cuestionarios a informantes clave. Entre las actividades más relevantes de educación ambiental realizadas por las ASADAS destacan la georreferenciación de manantiales, la reforestación, la formulación de proyectos, las capacitaciones, el manejo de residuos sólidos, las celebraciones y las giras de campo.

Palabras claves: protección; recurso hídrico; georreferenciación; reforestación; capacitación.

\section{ABSTRACT}

We investigate the Environmental Education process carried out by three Administrative Associations of Water Supply and Sewerage Systems (ASADAS, for its acronym in Spanish). We describe the EE activities carried out by these entities, which, in addition to supplying drinking water, must protect the hydric resource. The purpose of this publication is to inform about the role that ASADAS are playing both in hydric resource protection and in biodiversity conservation. This information is useful to improve what the studied ASADAS have done so far, and to promote environmental education in other associations. The study required documentary review, semi-structured interviews, and questionnaire application to key informants. Among the most relevant environmental education activities developed by the ASADAS are spring georeferencing, reforestation, training, special date celebrations, project formulation, solid waste management, protected areas damage reports, and field trips.

Keywords: protection; hydric resource; georeferencing; reforestation; training.

* Estudiante de la Escuela de Geografía de la Universidad de Costa Rica. Estudiante de Geografía, Universidad de Costa Rica, Costa Rica, minorva77@gmail.com

ID: https://orcid.org/0000-0002-3537-8658

* Investigador de la Escuela de Geografía de la Universidad de Costa Rica, mario.fernandezarce@ucr.ac.cr ID: http://orcid.org/0000-0003-3618-9995 


\section{Introducción}

La conservación y el uso sostenible de la biodiversidad forman parte del Plan Estratégico Institucional del SINAC 2010-2015. El plan pretende resguardar los ecosistemas, garantizar un marco jurídico para proteger y usar los recursos naturales, destacar los beneficios de los bienes, garantizar que las políticas de desarrollo prioricen la protección y el uso sostenible de los recursos naturales y generar tanto información como educación para los fines antes mencionados (SINAC, 2010).

Peña (2008) define la gestión integral del recurso hídrico (GIRH) como "un proceso que promueve el manejo y desarrollo coordinado del agua, la tierra y los recursos relacionados, con el fin de maximizar el bienestar social y económico resultante de manera equitativa sin comprometer la sustentabilidad de los ecosistemas vitales" (p. 10). La protección y manejo del recurso hídrico les compete a todos los miembros de la sociedad. La GIRH contribuye con lo anterior, con la administración sostenible y equilibrada del bien común y también con la coordinación del manejo del mismo en todos los sectores y grupos de interés. Implica la formulación de leyes y políticas que permitan tomar decisiones más equitativas y sostenibles y contempla evaluaciones, instrumentos económicos, sistemas de vigilancia (GWP, 2009) y aspectos sociales, económicos y ambientales.

En Costa Rica, existe el proyecto de ley denominado: Ley para la Gestión Integrada del Recurso Hídrico (Expediente N.o 17.742). La propuesta sobre la GIRH para Costa Rica se apoya en los principios de la Declaración de Dublín de 1992, a saber: 1- El agua es un recurso finito y vulnerable, esencial para sostener la vida, el desarrollo y el medio ambiente;
2- El aprovechamiento y la gestión del agua debe inspirarse en un planteamiento basado en la participación de los usuarios, los planificadores y los responsables de las decisiones a todos los niveles; 3- La mujer desempeña un papel fundamental en el abastecimiento, la gestión y la protección del agua y 4- El agua tiene un valor económico en todos sus diversos usos en competencia a los que se destina y debería reconocérsele como un bien económico (Agua.org.mx, 2021).

De acuerdo con la Carta de Nevada, UICN de 1970, citada por Alonso (2010), la educación ambiental se define de la siguiente forma:

[...] proceso de reconocer valores y clarificar conceptos, en el orden de desarrollar las destrezas y actitudes necesarias para comprender y apreciar las interrelaciones entre el hombre, su cultura y su entorno biofísico. La educación ambiental también implica la práctica en la toma de decisiones y en la autoformación de un código de conducta acerca de las cuestiones que afectan a la calidad ambiental. (p. 8)

Una definición más sencilla es la del SINAC (2010), a saber:

Es la educación orientada a enseñar cómo funcionan los ambientes naturales $y$, en particular, cómo los seres humanos pueden cuidar los ecosistemas para vivir de modo sostenible. Implica construir información, conocimientos, valores y actitudes, que son las herramientas fundamentales con las cuales actuar sobre los problemas ambientales. (p. 19)

Para Novo (1996) la educación ambiental es un movimiento ético y UNESCO (1977) la considera como la mejora de todas las relaciones psicológicas para lograr que el ser humano tenga conciencia del ambiente, se interese en él e impulse acciones para solucionar problemas ambientales. Guier et al. 
(2002) estiman que la educación ambiental debe tener como base el pensamiento crítico e innovador, en cualquier tiempo y lugar. Además es participativa, es decir, requiere contacto personal entre educador y educando en actividades como charlas, talleres, giras, proyectos y recolección de fondos para la solución de problemas específicos, entre otros (Brenes, 1994).

Se efectuó una investigación sobre el proceso en educación ambiental realizado por las ASADAS de Santiago de Palmares, Sabanilla, y Carrizal de Alajuela (figura 1), la cual requirió revisión bibliográfica exhaustiva y diseño de instrumentos para recolectar la información, tales como entrevista semiestructurada, grupo focal, observación no participante y encuesta. Por medio del trabajo de campo se obtuvieron datos relevantes para el estudio, los cuales fueron proporcionados por educadores ambientales del Área de Conservación Central (ACC) del SINAC, integrantes de las ASADAS, el director del ACC, así como otros funcionarios del SINAC, entre los que destaca un encargado del programa de recurso hídrico.

La información que se recabó fue sistematizada y analizada por medio de revisión documental y triangulación metodológica. La triangulación metodológica es una técnica que considera los datos generados por los

Figura 1

Mapa de ubicación

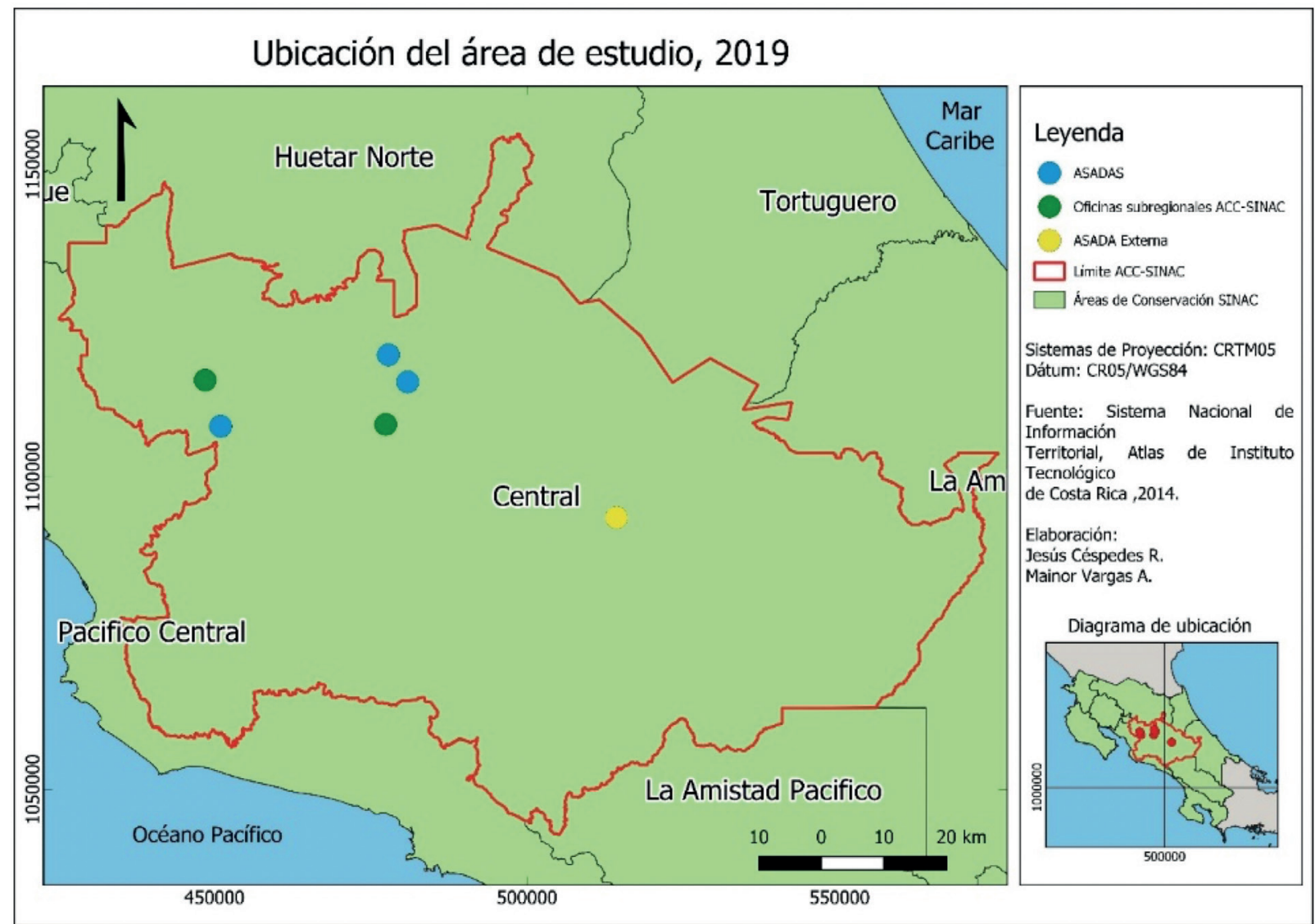

Nota. Elaborado por Jesús Céspedes y Mainor Vargas sistema con base en el Sistema Nacional de Información, Atlas del Instituto Tecnológico de Costa Rica (2014). 
diferentes métodos empleados para la producción de la información, destacando las coincidencias, similitudes, contrastes, complementariedad 0 ambigüedad entre los aportes realizados por los participantes; de esta forma, es posible realizar diversas interpretaciones sobre el tema que se desea analizar, cuyos resultados se pueden emplear como base para establecer conclusiones.

El estudio parte de la necesidad de conocer el grado de educación ambiental ejecutado por las ASADAS, dado su potencial como entes que velan por la protección del recurso hídrico y por la conservación de la naturaleza. Para bien de todas ellas y de toda la población del país, lo ideal es que las entidades citadas estén sumamente comprometidas con la educación ambiental. Todo trabajo excelente y valioso podrá ser útil para motivar, dar ejemplo y replicar. De ahí la importancia de recopilar información sobre las buenas prácticas de educación ambiental realizadas por las ASADAS.

El objetivo de la investigación fue conocer las actividades de educación ambiental ejecutadas por las tres ASADAS y dar a conocer los hallazgos. Se usarán los resultados como insumo para motivar a otras ASADAS y mejorar su trabajo. Si los resultados reprueban el trabajo en educación ambiental, entonces habrá una posibilidad para mejorar y redoblar esfuerzos. Si los resultados son satisfactorios, la réplica de las buenas prácticas podrá beneficiar a muchas otras asociaciones.

Para lograr el objetivo propuesto, se realizaron entrevistas semiestructuradas, encuestas, grupos focales y observación no participante. Las personas participantes seleccionadas fueron conocedoras del tema, como los funcionarios del ACC y los miembros de las
ASADAS. El trabajo de campo permitió recolectar la información que fue interpretada y analizada por medio de la triangulación metodológica.

El desarrollo de la investigación constató que los resultados del trabajo en educación ambiental son distintos para las tres ASADAS, lo cual depende de diversos factores entre los que destaca el contexto local y particularmente la población, incluyendo a los integrantes de las ASADAS con respecto al interés que muestran para involucrarse en el proceso; también se consideró, de forma paralela, el trabajo y la coordinación con oficinas subregional del SINAC como un factor determinante para los resultados obtenidos en la materia.

\section{Metodología}

La investigación, cuyo enfoque fue cualitativo, inició con una revisión documental que implicó la búsqueda y el análisis de información relacionada con educación ambiental en zonas aledañas a Quebradas (Brenes, 1994), capacitación para el uso racional del agua (Mayorga, 2012), empoderamiento de base comunitaria (Loaiza y Azofeifa, 2013) y participación comunitaria en gestión del recurso hídrico (Murcia y Vega, 2013). El trabajo Diseño de un sistema de monitoreo o seguimiento del proceso en educación ambiental desarrollado con ASADAS en el Área de Conservación Central del SINAC. Estudio de caso en las ASADAS de Santiago de Palmares, Sabanilla y Carrizal de Alajuela, 2018-2019 (Vargas, 2019), fue básico y esencial como referencia.

Para conocer la labor de las ASADAS en materia de educación ambiental se recurrió a entrevistas semiestructuradas, grupos focales (figura 2) y una encuesta dirigida a educadoras ambientales del SINAC que colaboran con 
las entidades estudiadas. Con el fin de complementar la colecta de información, se estableció comunicación vía correo electrónico, principalmente para aclarar dudas y ampliar los datos ya obtenidos. El trabajo de campo consistió en la aplicación de los instrumentos antes mencionados y las entrevistas, con el fin de conocer percepciones, valores, interpretaciones y experiencias de los funcionarios de las ASADAS y los educadores ambientales allegados a ellas sobre actividades de educación ambiental realizadas.

Figura 2

Grupo focal con la Junta Directiva de la ASADA de Sabanilla de Alajuela

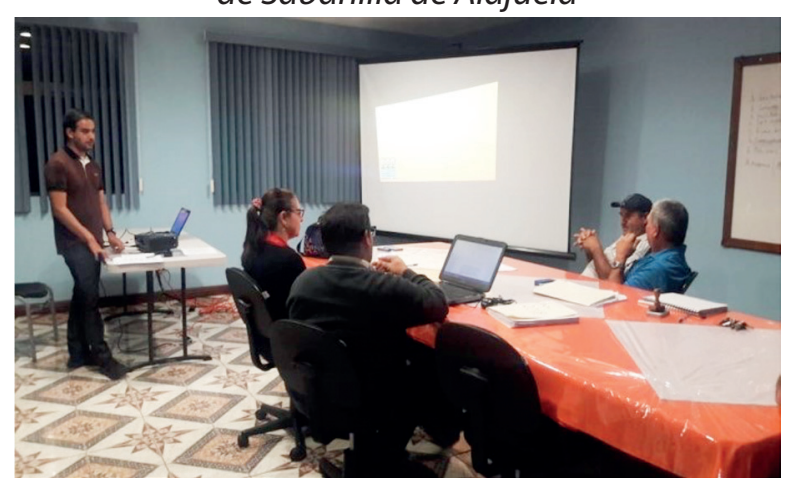

Nota. Fotografía de Mainor Vargas Araya, Sabanilla de Alajuela, 2018.

Una vez obtenidos los datos de campo se hizo una descripción de las actividades. Ello incluyó el ordenamiento y la interpretación de la información y de los testimonios manifestados por las y los participantes. Fue necesario comparar la información de las tres ASADAS para determinar cuáles actividades tenían en común.

\section{Resultados}

Como parte del trabajo que se realiza en las ASADAS, se han desarrollado actividades específicas como: georreferenciación de manantiales, reforestación, formulación y ejecución de proyectos, denuncias de acciones conflictivas, capacitaciones, manejo de residuos sólidos, giras de campo y celebración de fechas especiales relacionadas con temas ambientales y de recurso hídrico.

\section{Georreferenciación de manantiales}

Una de las actividades ha sido la georreferenciación de los manantiales con el fin de conocer su distribución espacial. La acción es de suma importancia para que las comunidades cercanas a los acueductos adquieran mayor interés por el agua, su uso, manejo y aprovechamiento. Se recorren las microcuencas para identificar los manantiales y obtener su ubicación, para luego elaborar mapas a partir del Sistema de Información Geográfica (SIG).

Las acciones sobre la distribución de los manantiales generan interés, dimensionan su importancia y permiten gestionar estrategias de conservación. Es importante ubicar geográficamente las fuentes hídricas a fin de crear una base de datos que sirva para la vigilancia de caudales, proponer estrategias de racionalización, optimizar el uso y dar sostenibilidad al servicio. De acuerdo con la investigación, la única ASADA de las tres estudiadas que hace esta labor es la de Sabanilla.

\section{Reforestación}

La vegetación ribereña contribuye con la habilidad de los ecosistemas de agua dulce para subsistir. Conforme disminuye la franja boscosa a lo largo de ríos y aumenta el área de pasturas, se incrementa la posibilidad de contaminación. El bosque retarda y reduce la escorrentía superficial, atrapa contaminantes, protege los acuíferos, aumenta la 
infiltración del agua superficial y, por lo anterior, la mejora de la calidad del agua (Cepeda y Navarro, 2010). Es necesario proteger el recurso hídrico, mantener su calidad y dar continuidad al suministro de agua potable. Para ello se deben crear zonas de protección con suficiente ancho de las franjas ribereñas y con adecuados usos en terrenos adyacentes, que garanticen el mantenimiento de las funciones ecológicas.

De lo anterior, se infiere la necesidad de reconocer las especies existentes a lo largo de los manantiales, que sirven para la protección del recurso hídrico. La reforestación de tales áreas requiere especies nativas, por lo que es necesario identificarlas. Por tanto, los miembros de las ASADAS estudiadas llevan a cabo actividades de reconocimiento de especies silvestres y reciben cursos en los que se les recomiendan especies que tanto ellos como miembros de la comunidad pueden usar para reforestar.

Las ASADAS de Sabanilla y Santiago de Palmares reforestan, para lo cual involucran a la población estudiantil de las escuelas y a personas voluntarias, algunas de las cuales llegan desde otras provincias, inclusive. De acuerdo con los datos recabados, algunas personas se ofrecen a colaborar con las campañas de reforestación. Los árboles los consiguen en instituciones como la Asociación de Seguridad y Embellecimiento de Carreteras Nacionales (ASECAN), asociación creada en 1974 para mantener, mejorar y promover el embellecimiento de los derechos de vía de las carreteras de Costa Rica, así también como de los aeropuertos del país, zonas turísticas, áreas tanto públicas como privadas (ASECAN, 2021).

Las especies donadas son aptas para la zona y se plantan en áreas de mantos acuíferos, en las fincas de los acueductos, principalmente cerca de las nacientes, con el fin de protegerlas y cuidar el recurso hídrico. En Santiago de Palmares se han hecho campañas de reforestación con niños de sexto año, quienes colocan una placa con su nombre en el árbol plantado. Algunos de los árboles ya son altos y frondosos, porque se plantaron hace aproximadamente ocho o nueve años. De igual manera, quienes los plantaron siguen participando en campañas ambientales, según indicó el administrador y presidente de la ASADA mencionada.

\section{Formulación y ejecución de proyectos}

Las ASADAS tienen que ejecutar proyectos o participar en ellos. Ha habido iniciativas a nivel nacional que las han involucrado y ellas mismas deben elaborar propuestas para construir su propia infraestructura de abastecimiento de agua y desarrollar proyectos socioambientales que promuevan prácticas sostenibles del recurso hídrico entre los usuarios, incluyendo medidas de adaptación basadas en los ecosistemas. Para ello es deseable una amplia participación comunitaria.

Uno de los primeros proyectos mencionados en el que participó la ASADA de Sabanilla es el denominado Removiendo Barreras para la Sostenibilidad del Sistema de Áreas Protegidas en Costa Rica, cuyo objetivo fundamental fue remover las principales barreras sistémicas e institucionales para la sostenibilidad del sistema de áreas protegidas de Costa Rica (Sosa, 2012), el cual fue liderado por el Programa de la Naciones Unidas para el Desarrollo (PNUD), en colaboración con el Ministerio de Ambiente y Energía de Costa Rica (MINAE) y el Sistema Nacional de Áreas de Conservación (SINAC). Funcionarios del SINAC se acercaron a 
la ASADA de Sabanilla (la única de las tres estudiadas) para desarrollar el proyecto, se enfatizó en el fortalecimiento de las habilidades de gestión de la ASADA y en desarrollar destrezas para ello. Se les inculcó a las personas participantes la idea de la organización comunal y se inició con un proceso de inducción para que ellos visualizaran su entidad como una organización base que puede hacer más cosas que llevar agua a otros. El dinero asignado se podía invertir en mejoras o protección de la naciente, pero se debía involucrar directamente a la comunidad (niños y adultos).

Se planteó un documento guía para el desarrollo del proyecto que incluyera cronograma, presupuesto y seguimiento. En esencia, se colocó malla alrededor de la naciente de la montañita y se inició una actividad de educación ambiental que incluyó la elaboración de afiches, banners, folletos educativos y giras para compartir las experiencias con otras ASADAS.

El Programa Sello de Calidad Sanitaria del Instituto Costarricense de Acueductos y Alcantarillados (AyA) ha sido acogido por la ASADA de Santiago de Palmares. El programa otorga, como incentivo, una bandera que se entrega anualmente como premio al esfuerzo, al trabajo y la dedicación de los diferentes entes operadores de acueductos, centros de salud, restaurantes, hoteles, centros de recreación y otros establecimientos para beneficio de los consumidores (Instituto Costarricense de Acueductos y Alcantarillados, 2021).

El AyA concede el premio a quien suministra agua de calidad potable en forma sostenible y en armonía con la naturaleza. La ASADA de Santiago de Palmares participa en ese programa desde el 2015 y para ello cuenta con la ayuda de la Promotora Ambiental Madre Verde (PRAMAVE). Para obtener el galardón debe entregar un informe en el que demuestra que cumple con los requisitos del programa, que está muy enfocada en el tema ambiental, que tiene conciencia ambiental; también deben aparecer charlas, plan de seguridad del agua, manejo de residuos y limpieza de ríos.

Los integrantes de las ASADAS indicaron que necesitan más capacitación para la gestión de proyectos socioambientales e inducción a los procesos que forman parte de tales proyectos. Por la falta de experiencia, tiempo y personal, ellos requieren acompañamiento de profesionales externos desde el inicio de las propuestas.

\section{Denuncias}

Los conflictos ambientales son un tema de mucha relevancia, se les dedican espacios como el Congreso Latinoamericano de Conflictos Ambientales (COLCA). Durante el COLCA (2016) se discutieron 48 casos de conflictos socioambientales entre los que destacan algunos relacionados con la conservación de la biodiversidad y el uso del recurso hídrico (Chacón, 2016). El recurso hídrico y el ambiente son elementos muy susceptibles a disputas y contiendas. Quienes los protegen y buscan el bien común se enfrentan a entes privados que buscan su propio interés. Por ello, los miembros de las ASADAS, en calidad de protectores del agua, tienen que prepararse para enfrentar eventuales conflictos y buscar soluciones satisfactorias.

Acciones que generan problemas son: cacería, extracción de flora y fauna, inadecuado manejo de residuos, deforestación, cambios de uso de suelo, invasión de áreas de protección, gestión inadecuada del recurso hídrico, mal manejo de cuencas, mal manejo del fuego, 
comercialización y trasiego de especies, acciones negativas de los visitantes y dueños de fincas dentro de las Áreas Silvestres Protegidas (ASP) y uso inadecuado de agroquímicos (SINAC, 2018). Los miembros de las ASADAS denuncian tala de árboles, remoción de terrenos cerca de nacientes y contaminación. "En una ocasión hubo remoción del suelo cerca de una naciente de la ASADA de Sabanilla, que dejó la malla protectora en el suelo y árboles prácticamente encima de la naciente, lo cual fue reportado y visto por funcionarios competentes pero la denuncia quedó en nada" (Junta Directiva de ASADA de Sabanilla, comunicación personal, octubre de 2018). La ASADA de Carrizal reportó que tienen conflicto con un refugio de animales denominado Territorio de Zaguates, el cual está a 200,5 metros de la fuente principal del acueducto, por lo que existe temor de contaminación. Como no se le da seguimiento a las denuncias, se desconoce el desenlace de ellas.

\section{Capacitaciones}

Las personas integrantes de las ASADAS necesitan capacitación en diversos temas pues requieren aprender de un amplio grupo de profesionales y apelan a la institucionalidad del país a fin de obtener el conocimiento básico deseado. Toda actividad formativa debe tener una intencionalidad educativa, es decir, ir más allá de la simple transferencia de conocimiento y generar autonomía, a fin de que las personas capacitadas puedan transferir conocimientos a la población local. Hasta el momento, el principal apoyo recibido por los funcionarios de las ASADAS es el de personas educadoras ambientales del SINAC.

Un evento destacable fue la dinámica sobre el transporte del agua en una cuenca hidrográfica. Fue una actividad lúdica en la que participaron miembros de las tres ASADAS estudiadas. Con los representantes se conformaron grupos de trabajo, se requirieron algunas cuerdas, sábanas, sillas, mesas y árboles. Se usó un recipiente con agua, atado con varias cuerdas sostenidas por los integrantes de cada uno de los grupos. Se simuló un recorrido por el parque y sus elementos, emulando lo que debe hacer el agua para rebasar los obstáculos que encuentra al atravesar la cuenca hidrográfica y llegar al mar.

En otra ocasión, un representante del Estado de la Nación expuso sobre conservación de áreas silvestres y los conflictos relacionados con el agua en otros lugares, destacando carencias y limitantes en varias partes del país. Lo anterior ayudó para que las personas participantes se dieran cuenta del valor del agua que disfrutan y a la vez la necesidad de abrir la mentalidad de los miembros de la comunidad para que valoren y protejan sus recursos hídricos.

Esencial es la participación de la comunidad en la educación ambiental, sobre todo de la población joven porque en ella se cifra la esperanza de que tanto la ASADA como su quehacer estará en sus manos en el futuro. Por ello, las y los funcionarios de las ASADAS van a las escuelas a impartir charlas, acompañados de algún funcionario del SINAC. Para esas actividades, la ASADA de Sabanilla ha usado libros para colorear que contienen información sobre conservación de los recursos naturales, en particular, la protección de nacientes. Se les enseña a las y los niños a cuidar y conservar el recurso, porque es agotable, y a evitar la contaminación de los ríos.

También se han impartido charlas a nivel comunal, para adultos, sobre las áreas de 
protección, en las que participan grupos organizados como la Policía y la Cruz Roja. La ASADA de Carrizal ofrece charlas de educación ambiental en la misma finca del acueducto y trabaja con jóvenes y miembros de la comunidad estudiantil de centros de educación preescolar y escuelas. La ASADA de Santiago de Palmares también participa en charlas en escuelas de las comunidades.

\section{Manejo de residuos sólidos}

La ASADA de Santiago de Palmares participa en el Programa Bandera Azul Ecológica, categoría Hogares Sostenibles y en el Programa Basura Cero. Para ello, realizan campañas de reciclaje una vez al mes con la ayuda de jóvenes voluntarios encargados de la separación y el reciclaje. La mayoría del voluntariado son muchachos que necesitan cubrir horas comunales. Paralelamente, se ejecutan campañas de limpieza de ríos y de calles. Lo anterior no solo ayuda a eliminar la basura de los ríos, sino a proteger la parte alta de la microcuenca donde se hallan las tomas de agua del acueducto, la reserva forestal, la zona de recarga de los acuíferos y las nacientes. La ASADA de Carrizal practica reciclaje y ejecuta la recolección de residuos sólidos.

\section{Giras}

Las giras de campo son una actividad relevante para quienes integran las ASADAS y también para los usuarios de las mismas. Durante las expediciones, se aprende de expertos sobre temas relativos a ecosistemas, recurso hídrico y manejo de conflictos. De los biólogos se adquieren conocimiento sobre especies nativas útiles para proteger el suelo, de los hidrólogos sobre dinámicas de fluidos, de los hidrogeólogos sobre las aguas subterráneas y de la fuerza pública sobre el delito que constituye la invasión de las zonas protegidas.

Los funcionarios del SINAC pueden mostrar el área de protección y las zonas invadidas. El saber citado puede ayudar a tomar mejores decisiones y hacer cumplir la ley. La articulación interinstitucional es necesaria para llevar a cabo las actividades porque entre más instituciones participen en una salida al campo, mayor será el aprendizaje de las personas que participen. En todas las ASADAS se ha realizado trabajo de campo. Los niños de las escuelas son llevados a las nacientes para que tomen conciencia de la importancia del agua y de su protección. También, los miembros de las ASADAS se visitan para conocer y comunicar buenas prácticas.

\section{Celebraciones}

En Sabanilla, celebran el Día de la Virgen de Lourdes que se le conoce como la Virgen del Agua. El evento es organizado por la ASADA e implica escoger la comunidad del distrito donde impartir la misa y dar un refrigerio que se cubre con dinero de la ASADA. La actividad ha sido muy aceptada, es en febrero y ha servido a la ASADA para proyectarse.

También celebran el Día Mundial del Agua, para ello escogen un pueblo y hacen la actividad en su escuela. La iniciativa comenzó en la escuela de San Luis de Sabanilla cuyo director abrió las puertas a la participación de toda la población. Los maestros se motivaron y se involucraron, al punto de grabar un video alusivo al agua y visitar la naciente de La Montañita, que se ubica detrás de dicha escuela, lo cual era desconocido para ellos. Además, se han hecho concursos de dibujo 
alusivos al tema del agua y los jueces han sido maestros de la misma institución.

Otro evento importante es la celebración de la Semana Ecológica en Carrizal y la Feria Ambiental organizada por un grupo denominado SULA, la cual involucra a jóvenes, centros de educación preescolar, escuelas y colegios. La participación es significativa y llega gente proveniente de muchos lugares. En la ASADA de Carrizal y en la de Sabanilla también celebran fechas especiales como el Día Mundial del Agua, el Día del Árbol, el Día Internacional de la Madre Tierra, entre otras (Vargas, 2019).

\section{Conclusiones}

Las ASADAS son aliadas estratégicas del MINAE para la protección del recurso hídrico y la conservación de la biodiversidad en el territorio nacional, especialmente en las zonas rurales, donde operan en mayor número; su valioso aporte es reconocido por las autoridades del Plan de Acción del Programa de Educación Ambiental del Área de Conservación Central (ACC) en el contexto del proceso en educación ambiental. Las asociaciones administradoras de los acueductos tienen potencial para proteger la biodiversidad y el recurso hídrico, pero su acción se ve limitada por los escasos recursos humanos y económicos.

La educación ambiental desarrollada en las ASADAS ha incluido las siguientes actividades: georreferenciación de manantiales, reforestación, formulación de proyectos, capacitaciones, denuncias, manejo de residuos sólidos y giras de campo.

El ACC contempla un conjunto de problemáticas socioambientales entre las que destacan la cacería, la extracción de flora y fauna, el inadecuado manejo de residuos, la deforestación, el cambios de uso de suelos, la invasión de áreas de protección, el manejo inadecuado del recurso hídrico, el mal manejo de cuencas, el mal manejo del fuego, el comercialización y trasiego de especies, las acciones negativas de los visitantes y dueños de fincas dentro de las ASP y el uso inadecuado de agroquímicos.

Las actividades educativas para los miembros de las ASADAS han sido ejecutadas por educadoras ambientales del SINAC, el Estado de la Nación, el INA y la Universidad Técnica Nacional, entre otras. Ellas requieren mayor participación de miembros de las ASADAS porque hasta el momento las personas capacitadas son voluntarios que apoyan a las asociaciones. Algunos temas se han abordado reiteradamente por lo que ha habido saturación de información al respecto.

Acercarse a los usuarios de las ASADAS por medio de la educación ambiental representa un reto, y más aún, su participación activa. El establecimiento de mejoras en este tema implica un trabajo articulado con el ACC-SINAC $\mathrm{y}$ otras instituciones $\mathrm{u}$ organizaciones tanto locales como nacionales.

Como parte de la proyección a la comunidad, se han realizado actividades formativas que involucran a la población de centros educativos de primaria y secundaria y a la sociedad civil. Se ha intentado participar a finqueros de las zonas, lo cual ha sido difícil hasta la fecha.

\section{Agradecimientos}

Los autores agradecen a las educadoras ambientales Ana Yancy Jiménez Cordero del Área de Conservación Central, Oficina San Ramón y María Rosa Ramírez Vargas, encargada de educación ambiental de la Oficina Subregional de Alajuela por la valiosa información brindada. Nuestra gratitud también a Kathia Soto, secretaria de la ASADA de Sabanilla de Alajuela por suministrar información útil a la investigación. 


\section{Referencias}

Agua.org.mx (2017). Declaración de Dublín Sobre el Agua y el DesarrolloSostenible.https://agua.org.mx/biblioteca/ declaracion-dublin-agua-desarrollo-sostenible/

Alonso Marcos, B. (2010). Historia de la Educación Ambiental. "La Educación Ambiental en el Siglo XX". Asociación Española de Educación ambiental (AEEA). http://ae-ea.es/wp-content/uploads/2016/06/ Historia-de-la-educacion-ambiental.pdf

ASECAN (Asociación de Seguridad y Embellecimiento de Carreteras Nacionales). (9 de noviembre de 2021). Descripción. https://yellow.place/es/ asecan-san-josé-costarica

Brenes, O. (1994). Intervención Comunitaria de Educación Ambiental No Formal con los pobladores de las zonas aledañas a la Quebrada Los Negritos. [Tesis de Licenciatura, Universidad de Costa Rica]. San José, Costa Rica, Ciudad Universitaria Rodrigo Facio.

Cepeda, C., Navarro, G. (2010). Protección del recurso hídrico en Costa Rica: propuesta para la reforma de los artículos 33 y 34 de la Ley Forestal. Turrialba, Costa Rica, CATIE. 93 p. Boletín Técnico n. 95. Colección Economía, Política y Gobernanza del Ordenamiento de Recursos Naturales n. 15.

Chacón, V. (22 de setiembre de 2016). Ecologistas denuncian aumento de conflictos ambientales. Semanario Universidad. https://semanariouniversidad.com/pais/ecologistas-denuncian-aumento-conflictos-ambientales/

Global Water Partnership (GWP, 2009). Manual para la Gestión Integrada de Recursos Hídricos en Cuencas. Empresa Gráfica Mosca. Londres, Reino Unido. http://www.rioc.org/IMG/pdf/RIOC GWP Manual para la gestion integrada.pdf

Guier, E., Rodríguez, M. y Zúñiga, M. (2002). Educación ambiental en Costa Rica. San José: Editorial de la Universidad Estatal a Distancia.

Instituto Costarricense de Acueductos y Alcantarillados. (21 de octubre de 2021). Programa Sello de Calidad Sanitaria. https://www.aya.go.cr/laboratorio/selloCalidad/Paginas/defaultaspx

Loaiza, E., Azofeifa, C. (2013). Fortalecimiento organizacional para el empoderamiento de la Base Comunitaria de la Microcuenca del río Purires. [Tesis de Licenciatura, Universidad de Costa Rica].
Mayorga, F. (2012). Propuesta de capacitación para el uso racional del servicio de agua potable de los usuarios del acueducto del centro de San José de Upala. [Tesis de Licenciatura, Universidad de Costa Rica].

Murcia, S., Vega, K. (2013). Participación comunitaria y gestión local en el manejo del recurso hídrico, en la ASADA de Tacares Sur de Grecia y en la ASADA de Carrillos Alto de Poás, provincia de Alajuela. [Tesis de Licenciatura, Universidad de Costa Rica].

Novo, M. (1996). La Educación ambiental formal y No Formal: dos sistemas complementarios. Revista Iberoamericana de Educación. n. 11, pp. 75-102. http://www.bio-nica.info/Biblioteca/Novo1996.pdf

Organización de las Naciones Unidas para la Educación, la Ciencia y la Cultura (UNESCO, 1977). Tendencias de la educación ambiental. París, Francia, 276 pp.

Peña, M. (2008). Gestión Integrada del Recurso Hídrico en la Legislación Costarricense. Editorial Investigaciones Jurídicas S. A. San José, Costa Rica.

Sistema Nacional de Áreas de Conservación (SINAC, 2010). Plan Estratégico Sistema Nacional de Áreas de Conservación-SINAC 2010-2015. San José, Costa Rica.http://www.sinac.go.cr/ES/docu/Planificacion/ Plan\%20Estrat\%C3\%A9gico\%20Institucional\%20 SINAC\%202010-2015.pdf.

Sistema Nacional de Áreas de Conservación. (SINAC, 2018). Plan de Acción del Programa de Educación ambiental del ACC 2017-2022. Equipo de Educadores Ambientales del ACC. Oficina Regional del Área de Conservación Central del SINAC, Santo Domingo de Heredia, Costa Rica.

Sosa, E., (2012).Informedela Auditoría del proyecto Removiendo Barreras para la Sostenibilidad del Sistema de Áreas Protegidas en Costa Rica. https://info.undp.org/ docs/pdc/Documents/CRI/00046871\%20-\%20 PROYECTO\%2OREMOVIENDO\%20BARRERAS\%20 2011\%20FINAL.pdf.

Vargas, M., (2019). Diseño de un sistema de monitoreo o seguimiento del proceso en Educación ambiental desarrollado con ASADAS en el Área de Conservación Central del SINAC. Estudio de caso en las ASADAS de Santiago de Palmares, Sabanilla y Carrizal de Alajuela, 2018-2019. [Tesis de Licenciatura, Universidad de Costa Rica]. 